\title{
HACIA UNA INTERPRETACIÓN \\ DE LA NACIONALIZACION DE LA INDUSTRIA \\ PETROLERA MEXICANA
}

RAÚl DELGADO WISE*

Como se sabe, el petroleo mexicano tuvo una destacada participación mundial en la segunda década del presente siglo, cuando las grandes compañias petroleras apenas iniciaban su escala expansionista. No sólo estuvo en la mira de ellas y fue objeto de una intensa explotación que lo llevó a figurar en los primeros planos de las exportaciones mundiales de dicho energético, sino que alrededor de él se escenificó una trascendental batalla entre las grandes corporaciones petroleras. Dos de ellas, la Standard Oil de Nueva Jersey (hoy Exxon) y la Royal Dutch Shell, fueron protagonistas de uno de los más significativos episodios de nuestra historia: la nacionalización de la industria petrolera. Este hecho, singular por la época en que se produce, estampa un sello propio al proceso mexicano. Se convierte en un parteaguas en el desarrollo de la industria petrolera del país, cuyo camino se apart6 en múltiples sentidos de aquel seguido por el grueso de los miembros de la OPEP, pero que no deja de estar vinculado a los sucesos que se desarrollan en el mercado mundial.

El proposito de estas notas es precisamente el de aportar algunos elementos tendientes a esclarecer los entretelones del proceso que desembocó en la nacionalización de esta industria y que marco su derrotero hasta la irrupción del boom petrolero reciente. Más que nada nos interesa aclarar algunos aspectos decisivos de dicho proceso

* Profesor investigador, maestría en Ciencias Políticas, Facultad de Derecho, Universidad Autónoma de Zacatecas. 
que encuentran su explicación en la vía de crecimiento que a lo largo de ese periodo sigue la industria petrolera internacional: la secuencia ascendente de la renta diferencial.

\section{Los antecedentes}

Para el proposito planteado, los hechos significativos comienzan a principios de este siglo. Es entonces cuando dos constructores extranjeros de los ferrocarriles, uno inglés y otro norteamericano, se plantean el proyecto de explotar comercialmente nuestro petroleo. Se inicia así una etapa de intensa explotación y resonantes des. cubrimientos; una etapa signada por una desenfrenada carrera por la conquista de concesiones en la que ambos contratistas se ven enfrascados.

En este proceso, el primer logro importante se lo adjudicó el norteamericano Edward L. Dohney al descubrir importantes yacimientos en lo que después se conocería como la Faja de Oro. La participación del que fuera contratista del tramo férreo Tampico-San Luis Potosí sería decisiva. Dos compañías fundadas por él, la Mexican Petroleum Company y su subsidiaria, la Huasteca Petroleum, hicieron historia al convertirse, en 1904, en las primeras empresas del pais en producir petroleo comercialmente. ${ }^{1}$ Ambas empresas fueron, además, las que concentraron la mayor parte del capital norteamericano invertido en el ramo.

La representación inglesa, a cargo del contratista del Ferrocarril del Istmo, Weetman D. Pearson (Lord Cowdray, desde 1911), no corrio con la misma suerte inicial de Dohney. Tras una larga cadena de fra. casos y vicisitudes, la suerte del súbdito de la Corona británica cam. bió al entrar 1910. Irónicamente, sus mayores éxitos vendrían de donde menos los había esperado: el norte de Tuxpan. ${ }^{2}$ Un año antes, el contratista inglés registró la compañía El Águila, la cual, después

${ }^{1}$ Esperanza Durán, "El petróleo mexicano en la primera guerra mundial", Miguel S. Wionczek, Energia en Mexicu. Ensayos sobre el pasado y el presente, COLMEX. México, 1982, pp, 57 y 58.

2 Ibid., p. 59. 
de haber tenido un inicio incierto, lograría colocarse en el primer plano de la producción nacional. Así, al finalizar 1910, dicha compañía se erigía ya como la principal productora del país, con $58 \%$ de la producción total. ${ }^{3} \mathrm{Y}$ este sitio lo mantendría hasta el momento de la expropiación, cuando había logrado conjuntar $70.6 \%$ de los bienes y servicios de la industria nacionalizada. ${ }^{4}$

Pero los contratistas ferroviarios sólo figurarian como el primer eslabón del proceso de explotación petrolera que en este periodo toma lugar en México. Intereses más poderosos muy pronto entrarian en escena. Capitalizando el camino allanado por sus coterráneos, las grandes compañias petroleras no tardaron mucho en hacer presencia directa en el país. En representación de los intereses imperiales del otro lado del Atlántico, la Royal Dutch Shell inicia operaciones en 1912, y hacia 1919 había adquirido control pleno de la mayor empresa en nuestro territorio: El Águila. ${ }^{5}$ Por su parte, del lado norteamericano, destacó la presencia de la Standard Oil de Nueva Jersey. Después de largo tiempo de haber intentado penetrar en nuestro país, ${ }^{6}$ la poderosa compañía logra, por fin, en 1925 , adquirir el

\section{${ }^{3}$ Ibid., p. 60.}

4 Miguel Alemán, La verdad del petróleo en México, 3a. ed., Grijalbo, México, 1977. p. 535.

5 Enrique Ruiz Garcí, La estrategia mundial del petróleo, Nueva imagen, México, 1982 , p. 70.

6 Hay al menos dos intentos de penetración de la Standard Oil que merecen ser mencionados aquí. El primero, aunque no es propiamente una tentativa de explotar el petróleo mexicano, es digno de senalarse por su importancia en la distribución y refi. nación del mineral. Data de fines del siglo pasado e involucra a otro personaje sobresaliente de la historia de nuestros hidrocarburos: Henry Clay Pierce. Se trata, concretamente de la participación de la empresa de Rockefeller con $65 \%$ de las acciones del que fuera el principal conglomerado petrolero del país hasta 1900: la Waters Pierce Oil Company. Dicha companí, dirigida por Pierce, detentaba el monopolio de la distribución y refinación del crudo importado de los Estados Unidos. A pesar de su dominio en las acciones, la Standard Oil nunca pudo impulsar su política en México debido al manejo independiente que Pierce daba a la companía. Frente a ello, tras infructuosas tentativas de adquirir las acciones de Pierce y por un mandato de la Suprema Corte de los Estados Unidos, se vio forzada a dar marcha atrás en su tenta. tiva y a vender su parte mayoritaria de las acciones a aquél. En su evolución posterior, la empresa de Pierce conservaría su independencia y se integraría al grupo Sinclair. Este se ganaría el tercer sitio en la explotación petrolera nacional y desempenaría, como 
paquete de acciones de Dohney en la Mexican Petroleum y la Huasteca Petroleum Company. ${ }^{7}$

La disputa por el petróleo en México cobraba así nuevas dimen. siones. Sus protagonistas habian cambiado y la lucha se insertaba en aquellas que libraban las grandeas corporaciones del petróleo en su escalada mundial. Los acontecimientos que se sucederían en el país estarían de una u otra manera relacionados con este hecho.

Como parte de la contienda por nuestros hidrocarburos, escenifica. da por los productores ferroviarios, primero, $\mathbf{y}$ por las grandes corporaciones, después, sobresale una característica del tipo de crecimien. to asumido por la naciente industria petrolera del pais: su orientación hacia el exterior. En torno a ello, que, por lo demás, concuerda con la forma de crecimiento general que en ese entonces imperaba en México, es como se define la dirección básica del proceso productivo. La organización entera de la producción de la época giraba alrededor del eficaz cumplimiento de ese propósito. Es por ello que las refi. nerias que se abrieron en ese tiempo se ubicaron en las zonas portua. rias. De aqui también que para la transportación de nuestro petroleo a Europa, Lord Cowdray, propietario de El Águila, haya hecho cons. truir la flota de buques-tanque más grande y eficiente del mundo de aquel entonces ${ }^{8}$ En ello estriba, asimismo, el hecho de que solo una proporción relativamente pequeña de la producción se haya desti. nado al mercado interno (a excepción de los años próximos a la nacionalización en los que, como veremos enseguida, la producción se encontraba en plena decadencia). Lo propio puede decirse de la poli. tica de precios de las compañas, que fijaba los internos muy por encima de los internacionales. ${ }^{9}$ Incluso, el que hayan sido precisa.

veremos más adelante, un papel importante en el proceso que sigue al decreto de nacionalización. Véase: Esperanza Durán, op. cit., p. 57. El otro intento frustrado de penetración que queriamos senalar fue cuando la Standard Oil pretendi6, en 1913 , adquirir las acciones de El Águila. Véase Lorenzo Meyer, "Los petróleos británicos, el nacionalismo mexicano y el gobierno de Su Majestad británica (1901-1947)", Miguel S. Wionczek, op. cit., p. 19.

${ }^{7}$ Enrique Ruiz García, op. cit., p. 68.

${ }^{8}$ Esperanza Durán, op. cit., p. 60.

9 -El Águila, según extensa documentación probatoria, vendía su gasolina en México a un precio $134.43 \%$ más alto que fuera del país..." (Enrique Ruiz García, op. cit., p. 69). 
mente los constructores de los ferrocarriles quienes iniciaran la explotación petrolera en el país, encuentra una clara relación con la característica referida. Ello, como bien lo puntualiza Ruiz García, adquiere significacion "...en el cuadro de una estrategia de transportes que hacía posible la integración de México en el mercado internacional de minerales..." $10 \mathrm{El}$ exterior aparece así como una dimensión privilegiada del análisis de la industria petrolera del país en esta su primera etapa.

Dentro de este marco general, en el que se define el carácter de la industria hasta el momento de la nacionalización, la producción del petróleo muestra un comportamiento dispar y contrastante. Atraviesa, como claramente se desprende del cuadro 1, por dos grandes fases.

En la primera, que se prolonga hasta 1921, el rasgo distintivo lo constituye la aceleración con la que crece la producción. Aquí se ubica uno de los periodos más exitosos de nuestra industria: aquel que inicia con la Revolución Mexicana y que llega a situar al país en los primeros planos de la producción mundial.

Curiosamente, el movimiento armado de 1910 no tiene ningún efecto pernicioso sobre el ritmo de explotación que, por el contrario, llego a acelerarse sustancialmente. Durante este lapso, el elevado ritmo de crecimiento de la producción, a una tasa anual de $43.5 \%$ (casi dos veces más explosivo que en el boom reciente), hizo posible que nuestro país se colocara en el tercer lugar de la producción mundial en 1911 - después de Estados Unidos y Rusia-y que alcan. zara el segundo lugar $\rightarrow$ sólo después de los Estados Unidos-en 1918.11

La segunda fase, que va de 1922 a 1938, constituye en gran medida la sombra de la anterior. El comportamiento que en ella sigue la producción resulta polar al que había mostrado hasta entonces. Tras la vigorosa cuesta arriba de la fase precedente, que le había permitido alcanzar un máximo de producción de 193397586 barriles en 1921 ,

${ }^{10}$ Ibid., p. 64.

11 Véase: Samuel I. del Villar, "Estado y petroleo en México: experiencias y perspectivas", Foro internacional, vol. XX, núm. 77 (jul-sep), 1979, p. 119 y Esperanza Durán, op. cit., p. 62. 
CUADRO 1

MÉXICO: EVOLUCIÓN DE LA PRODUCCIÓN PETROLERA ANTERIOR AL AUGE DE LOS SETENTA

\begin{tabular}{|c|c|c|c|}
\hline \multicolumn{2}{|c|}{ Antes de la nacionalización } & \multicolumn{2}{|c|}{ Después de la nacionalización } \\
\hline año & barriles & año & barriles \\
\hline 1901 & 10334 & 1938 & 38818213 \\
\hline 1902 & 40181 & 1939 & 43306543 \\
\hline 1903 & 75335 & 1940 & 44448191 \\
\hline 1904 & 125561 & 1941 & 43385822 \\
\hline 1905 & 251122 & 1942 & 35148633 \\
\hline 1906 & 502238 & 1943 & 35458894 \\
\hline 1907 & 1004475 & 1944 & 38503348 \\
\hline 1908 & 3931049 & 1945 & 43877430 \\
\hline 1909 & 2712091 & 1946 & 49532888 \\
\hline 1910 & 3632192 & 1947 & 57117911 \\
\hline 1911 & 12546286 & 1948 & 59773247 \\
\hline 1912 & 16549619 & 1949 & 63226983 \\
\hline 1913 & 25682957 & 1950 & 73881472 \\
\hline 1914 & 26221783 & 1951 & 78779552 \\
\hline 1915 & 32893429 & 1952 & 78906528 \\
\hline 1916 & 40545070 & 1953 & 74097590 \\
\hline 1917 & 55292767 & 1954 & 85230204 \\
\hline 1918 & 63828322 & 1955 & 91370125 \\
\hline 1919 & 87072954 & 1956 & 94370125 \\
\hline 1920 & 157068678 & 1957 & 92197297 \\
\hline 1921 & 193397586 & 1958 & 100641405 \\
\hline 1922 & 182278457 & 1959 & 105758471 \\
\hline 1923 & 149584855 & 1960 & 108771592 \\
\hline 1924 & 139678293 & 1961 & 116825375 \\
\hline 1925 & 115514698 & 1962 & 121792941 \\
\hline 1926 & 90420970 & 1963 & 125982207 \\
\hline 1927 & 64121140 & 1964 & 129503502 \\
\hline 1928 & 50150610 & 1965 & 132141019 \\
\hline 1929 & 44687877 & 1966 & 135021014 \\
\hline 1930 & 39529901 & 1967 & 127284625 \\
\hline 1931 & 33038854 & 1968 & 139293125 \\
\hline 1932 & 32805495 & 1969 & 146070810 \\
\hline 1933 & 34000828 & 1970 & 155544750 \\
\hline 1934 & 38172513 & 1971 & 154523115 \\
\hline 1095 & 40240864 & 1972 & 361253640 \\
\hline 1336 & 41027915 & 1973 & 160637825 \\
\hline \multirow[t]{3}{*}{1937} & 46906650 & 1974 & is7 445 c20 \\
\hline & & 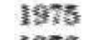 & 248 ing sats \\
\hline & & 1376 & 285364325 \\
\hline
\end{tabular}

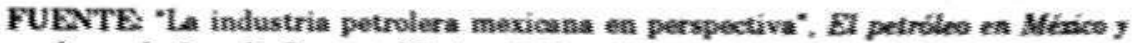

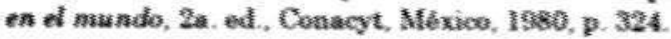


se sucede un rápido desplome de la misma hasta un mínimo de 32805495 barriles en 1932, esto es, casi una sexta parte del nivel alcanzado en 1921. Aunque esta violenta caída se detiene en 1932, los niveles de la producción no llegan a recuperarse y se mantienen más bien bajos. Cuando se produce la nacionalización, éstos apenas lograban rebasar el mínimo de 1932, con una producción de 38818213 barriles.

A pesar de su obvia significación económica, el esclarecimiento de las causas que provocan la caída descrita es algo que no ha recibido la atención que amerita en la literatura. En las contadas ocasiones en que se ha abordado, su tratamiento ha sido incompleto, en tanto que omite la consideración de un aspecto decisivo del contexto en que se inscribe el caso mexicano, a saber: la estrategia de crecimiento de las grandes corporaciones petroleras. ${ }^{12}$ No se trata simplemente de la omisión de un aspecto complementario de la temática, sino -como tendremos oportunidad de constatar- del aspecto clave de la explicación, sin el cual resulta imposible el cabal entendimiento del proceso de nacionalización, así como de las posiciones guardadas por los consorcios petroleros en el conflicto que sigue al acto expropiatorio.

Desde nuestro punto de vista, y como advertíamos en un principio, el elemento decisivo para explicar el comportamiento de la industria petrolera internacional durante el periodo en cuestion consiste en

12 Entre las escasas referencias a esta caída de la producción, lo más comán es atribuirla a la falta de garantías para realizar nuevas exploraciones, derivadas, tanto de la inestable situación política del pais, como del articulo 27 de la Constitución de 1917, que eliminaba el derecho de propiedad a los consorcios petroleros sobre el sub. suelo mexicano. Aunque no podemos negar la incidencia de tales factores, no es en ellos, como veremos, donde gravita la explicación del fenómeno. Seguir ese orden explicativo ha conducido, en nuestca opinión, a interpretaciones de la nacionalización cargadas de subjetivismo, en las que destaca la "mala fe" de las companias extranjeras exacerbando el nacionalismo mexicano.

Mencion aparte merece el punto de vista de la Comisión de Peritos encargada de rendir un informe sobre las companías petroleras en el conflicto obreropatronal que antecede al acto expropiatorio y en la que figuraba Jesús Silva Herzog. Dicha comisión, aunque todavía sin la claridad suficiente, percibe el punto cuando senala: "La disminu. ción petrolera en México se debe al agotamiento de los yacimientos, principalmente a los de la Faja de Oro; a la falta también de nuevas e intensas exploraciones para des. cubrir otros campos, y posiblemente a la política de las compañías petroleras" (Miguel Alemán, op. cit., p. 221; subrayado nuestro). 
ubicar el principio organizador de la estrategia de crecimiento de las grandes corporaciones en el criterio dictado por la renta diferencial en su secuencia ascendente. ${ }^{13} \mathrm{El}$ avance por esta vía hacia yacimientos con cualidades "naturales", cada vez más ventajosas, procurando el incremento progresivo de la demanda y vigilando celosamente que el ritmo de explotación no llegase a desbordarla, constituía el pun. tal del proceso expansionista seguido por las grandes corporaciones. Los acuerdos monopblicos que suscribieron en $1928^{14}$ no tenían otro proposito que ése: garantizar el disfrute de las rentas diferenciales que los yacimientos recién descubiertos en Medio Oriente posibili. taban.

Como es logico suponer, el proceso mexicano, fuertemente entre. lazado al proceso expansivo de la industria petrolera internacional, no podía permanecer ajeno a esos mismos principios rectores. Debía sujetarse a ellos y eso fue lo que efectivamente ocurrió. La pauta de ello nos la aporta la cualidad del crudo descubierto en nuestro territo. rio. Ésta resultaba comparativamente más ventajosa que la corres. pondiente al crudo disponible en la Unión Americana, como claramente se desprende del siguiente dictamen pericial realizado a las empresas extranjeras poco antes de la nacionalizacion:

El porciento de utilidad en relación con el capital invertido no amortizado de las empresas petroleras demanadadas excepción hecha de la Mexicana Gulf, fue en promedio, en los anos 1934 a 1936, de $16.82 \%$ [ ... ] Las principales empresas petroleras establecidas en los Estados Unidos obtuvieron en el ano de 1935 una utilidad, en relación con el capital invertido, de $6.13 \% .15$

13 Para un desarrollo térico de este criterio véase el capitulo XXXIX de El Capital de Karl Marx (libro III, vol. VIII, Siglo XXI, México, 1981).

14 Nos referimos al acuerdo red line firmado el 31 de julio de 1928 y al acuerdo de Achnacarry firmado el 17 de septiembre del mismo ano. El primero establecía una zona de operaciones comunes en Medio Oriente, al tiempo que el segundo marcaba ciertas pautas para garantizar el avance de los firmantes por la secuencia ascendente, asegurándoles el disfrute de jugosas rentas diferenciales.

${ }^{15}$ Miguel Alemán, op. cit. p. 223. 
De aquí se infiere una relación aproximada de casi 3 a 1 entre los respectivos beneficios del crudo mexicano y el norteamericano. No es extraño, por consiguiente, el interés puesto en nuestro petroleo por las grandes corporaciones. Y lo es menos aún si consideramos, por un lado, que al tiempo en que se expande aceleradamente la producción del país, existían muy pocos antecedentes del potencial que tendrían zonas como Medio Oriente y Venezuela. Todo sugiere que en ese entonces, independientemente del conflicto armado que aquí se vivía y de sus obvios inconvenientes para las compañias, el pais ofrecía una de las mejores alternativas del momento para la captación de rentas diferenciales. Por otro lado, nada parece indicar que durante esta fase de crecimiento existieran problemas significativos en relación con la demanda. Entre otras cosas, los requerimientos extraordinarios de los hidrocarburos suscitados por la Primera Guerra Mundial (la primera guerra motorizada en la historia) y el papel crucial que al respecto desempeño el petroleo mexicano ${ }^{16}$ debieron haber repercutido favorablemente para ello.

La violenta caída que a partir de 1922 sufre nuestra producción petrolera no se aparta en absoluto de esta misma línea de interpretación. Encuentra en ella una amplia coherencia que se manifiesta, como veremos enseguida, en la compatibilidad del fenómeno con el criterio regulador de la renta diferencial en su secuencia ascendente.

Dos aspectos de la expansión global de la industria petrolera resultan determinantes en este sentido. El primero tiene que ver con el inicio de grandes descubrimientos de campos petroleros gigantes en el Medio Oriente (que comienza precisamente un año antes de que se iniciara el desplome de nuestra produccion) ${ }^{17}$ y en Venezuela (cuya producción se acelera sensiblemente durante este lapso). ${ }^{18}$ Lo determinante en ambos casos es que las cualidades "naturales" de dichos campos superaban ampliamente a las de los yacimientos que se conocian en nuestro pais:

16 Esperanza Durán, op. cil., pp. 62-75.

${ }^{17}$ Richard Nehring. "Los campos petroleros gigantes y los recursos mundiales", El petróleo en México y en el mundo, op. cit., p. 43.

18 De no haber prácticamente figurado en el mapa petrolero en 1920, pudo superar a México en 1928. 
1. En cuanto a rendimientos, con las reservas de los datos disponibles, Medio Oriente y Venezuela guardaban una relación aproximada de poco más de 10 a 1 y de 4 a 1 con referencia al crudo norteamericano; ${ }^{19}$ miestras que México, como acabamos de ver, mantenía una relación de poco menos de 3 a 1.

2. En calidad (densidad, contenido de azufre y pureza), las desven. tajas comparativas del crudo mexicano que se explotaba en ese entonces se hacen evidentes, al haber sido éste, en su mayor parte, crudo pesado. ${ }^{20}$

3. En localización, la expansión de los mercados de Europa y Japón concedian ventajas relativas a los campos situados en el Medio Oriente.

Si a lo anterior agregamos las dificultades y trabas que (en la optica de las grandes corporaciones) el gobierno posrevolucionario les interponía y todo lo contrapesamos con las facilidades y garantías que otros Estados les concedían, la balanza general resulta altamente desfavorable para nuestro petroleo. El interés por el mismo había sufrido un vuelco radical y las perspectivas globales de expansión de la industria petrolera había dejado de apuntar hacia México.

La otra pinza de la explicación se relaciona con el comportamiento que sigue la demanda de hidrocarburos. Al respecto, es importante tener presente que la caída de la producción se inicia en la antesala del pacto monopólico suscrito por las grandes corporaciones petroleras en 1928. Se trata de una época de fuertes convulsiones en el mercado que entorpecían el avance por la línea ascendente; una época en que la riqueza de los nuevos descubrimientos y su enorme potencial tropezaban con el ritmo relativamente pausado al que crecía la demanda. Esta situación es advertida por Miguel Alemán, cuando, narrando las vísperas del colapso de la economía mundial de 1929, señala: “el primer aviso proviene del petróleo. La expansión de

19 Relación basada en datos proporcionados por G. B. Zorzoli, El dilema energético, H. Blume Ediciones, Madrid, 1981, p. 88 y Marcial Rubio Correa, OPEP. Imperialismo y Tercer mundo, Centro de Estudios y Promoción de Desarrollo, DESCD, Perú, 1976, p. 27.

20 "La industria petrolera mexicana en perspectiva", El petróleo en México y en el mundo, Conacyt, 2a ed., México, 1980, p. 321. 
la industria de hidrocarburos en Venezuela y Medio Oriente comienza a sobrepasar la demanda de los mercados internacionales. En México, el problema se ha puesto de presente desde la administración del Presidente Calles". ${ }^{21}$

La significación de este hecho se realza si consideramos que fue precisamente durante el régimen callista (1924-1928), cuando la producción petrolera del país experimentó su caída más drástica.

Los dos aspectos que hemos introducido confieren un significado preciso a la cuestión del desplome que desde 1922 experimenta la producción en México. En ellos, encuentra soporte nuestra tesis acerca de su compatibilidad con los principios que rigen el crecimiento global de la industria petrolera, esto es, con los postulados de la renta diferencial en su secuencia ascendente. Es así como la superioridad relativa del petroleo de Medio Oriente y Venezuela, por una parte, y el desbordamiento de la demanda que la expansión hacia esas zonas iba provocando, por la otra, se convierten en los ejes vertebrales para explicar la caída de la producción mexicana, expresan las causas de fondo de que el petróleo de nuestro pais cayera del pedestal en que se habia logrado situar al correr de la Primera Guerra Mundial. Asimismo, explican las razones del cambio en la política de inversiones de los grandes consorcios petroleros hacia México, que prácticamente se llegan a paralizar (en contraste con el enorme caudal de recursos que canalizan hacia las nuevas y más ricas zonas productoras). Esto a su vez, aunado al apetito voraz de las empresas instaladas en nuestro territorio que intentaban exprimir al máximo sus inversiones previas, explica el rápido agotamiento de los campos existentes y el consecuente desplome de la producción petrolera del pais.

21 Miguel Alemán, op. cit., p. 173. Más todavía, a la apreciación de Alemán acerca del desbordamiento de la demanda por los nuevos campos en operación en Venezuela y Medio Oriente habría que agregar la oferta de petrbleo barato proveniente de Rusia. Véase: Michael B. Storff, Oil, war and american security. The search for a national policy on foreign oil, 1941-1947, Yale University Press, New Haven, 1980, p. 8. 


\section{El proceso de expropiación}

Claramente, el viraje que se produce en la política de los consorcios internacionales hacia nuestros recursos petroliferos resulta alta. mente desquiciante para la economía del país. Se trata, empero, de un cambio que stricto sensu no se aparta de la misma estrategia expansionista que pocos años antes había sustentado una política diametralmente opuesta hacia nuestros hidrocarburos. El afianzamiento de dicha línea estratégica, que tiene lugar con la firma de los acuerdos de 1928, no afecta para nada la nueva orientacion; por el contrario, ella tiende a ser conducida por el mismo sendero hasta las áltimas consecuencias. Todo esto no hace sino proyectar una con. tradicción de fondos entre los intereses del capital petrolero y el crecimiento económico nacional. Si para uno el cambio de política signifi. caba ampliar sus perspectivas de lucro, para el otro implicaba desnudar el saqueo y la limitación de perspectivas de que estaban siendo objeto. Dicha situación explosiva estaba destinada a enfrentar, como de hecho lo hizo, al Estado mexicano con los grandes consorcios petroleros. Tal es, en nuestra opinión, el trasfondo de la serie de conflictos ya conocidos que desembocó en la expropiación de las empresas extranjeras que operaban en nuestro territorio, decretada el 18 de marzo de 1938.

Con todo, la nacionalización no era un resultado al que hubiesen querido llegar las empresas extranjeras. Y no lo era, no tanto porque nuestros hidrocarburos figuraran en sus planes de expansion de corto y mediano plazo (como se desprende del hecho de que con antelación a la nacionalización hayan dejado de invertir en nuevas explo. raciones), sino porque objetivamente el acto expropiatorio constituía una amenaza para su imperio. La posibilidad de que la nacionali. zación mexicana tuviera éxito y de que su ejemplo cundiera en otras latitudes era algo que no podian permitir. Habia que hacer de nuestro caso un verdadero escarmiento. Fue así como las compañías petroleras, con el respaldo inicial de sus respectivos gobiernos, organizaron un severo boicot contra México y especialmente contra la com. pañía petrolera nacional emergida del acto expropiatorio: Petroleos Mexicanos (Pemex). Entre otras cosas, el boicot abarcaba las siguientes acciones: 
i) bloqueo para que no pudiese colocar su petróleo en el mercado mundial; ii) interrupción de los abastecimientos de materias primas, refacciones y equipos que requería importar la industria petrolera mexicana; iii) presion para que las companias navieras de otros paises se negaran a transportar el petroleo mexicano; iv) acciones legales para embargar el petróleo que México lograba exportar a otras naciones; v) retiro masivo de los depósitos bancarios que las empresas extranjeras mantenían en México; vi) boicot a las exportaciones de otros productos mexicanos e, incluso, vii) incitacion de conflictos internos para alterar la situación política del pais. 22

Si en un principio, el boicot cobro mucha fuerza aglutinando los intereses de todas las corporaciones petroleras expropiadas, grandes y pequeñas, contando, además, con el apoyo de los gobiernos norteamericano e inglés, así como con el de algunos otros que se les sumaron, a medida que el conflicto se fue desarrollando, los intereses en pugna se fueron diferenciando $y$ el cerco tendido contra nuestro pais fue paulatinamente levantándose.

La mayor dureza en el conflicto fue mantenida por las grandes corporaciones petroleras. En el interés de ellas estaba hacer que Pemex fracasara y que la nacionalización diera marcha atrás. Hacia ello cargaron sus baterías. Su táctica, implementada una y otra vez a lo largo del dificil proceso negociador, consistio en obstruir cualquer arreglo tendiente a fijar el monto de la indemnización. De no darse el arreglo que buscaban, preferian mantener empantanadas esas negociaciones y prolongar en todo lo posible la indefinición reinante. Pensaban que el tiempo constituía su mejor aliado, no solo porque en sus planes de corto y mediano plazo el petróleo mexicano era básica. mente redundante, sino, además, porque preveían que sin su apoyo administrativo, financiero y técnico, el fracaso de Pemex estaría garantizado. Así se explica la cadena de reveses que siguieron a los intentos de negociación con el gobierno mexicano. ${ }^{23}$

22 Jaime Corredor Esnaola, "El petróleo en México", El mercado de valores, ano LXI, núm. 8 (febrero 23), 1981, p. 210.

${ }^{23}$ Entre las infructuosas tentativas de negociación, donde se proyecta nítidamente la táctica de los consorcios, cabe mencionar aquellas que se llevaron a cabo en la Ciudad de México y Saltillo entre el abogado Richberg y el presidente Cárdenas. Vúase: Miguel Alemán, op. cit., pp. 433-435. 
Si la táctica negociadora de las grandes corporaciones tenía senti. do en su estrategia global, no era así tanto para las empresas independientes. Sus intereses no estaban reflejados en el pacto monopo. lico suscrito en 1928 y, por lo mismo, no se correspondían con el interés hegemónico que se desprende de la estrategia de expansion global concertada por las grandes corporaciones. Por este motivo, la principal empresa independiente del país, el grupo Sinclair, asumio una posición distinta en el conflicto y decidió negociar por cuenta propia con el gobierno mexicano. Se convertiría de esta manera en la primera empresa del país en llegar a un acuerdo acerca del monto de la indemnización en mayo de $1940 .{ }^{24}$ Con esta histórica decision, que implicó romper filas y debilitar la posición de las grandes compañias, el grupo Sinclair no hacía sino proyectar sus propios intereses y las divergencias que al respecto tenía con dichas empresas.

No obstante que el caso Sinclair presagiaba el desenlace que final. mente tendría el conflicto, era apenas un primer eslabón del proceso. De mayor peso específico en él resultaría, sin duda, la posición de las dos grandes potencias involucradas directamente: Inglaterra y Estados Unidos. Ambas, no obstante que habian coincidido en su postura inicial, muy rápidamente se distanciaron y extremaron sus posi. ciones. Mientras que la parte británica se mostró inflexible e intolerante respecto a la nacionalización, al grado de llegar al rompimiento de relaciones con nuestro país, la postura norteamericana manifestó ser más flexible. El ejecutivo de los Estados Unidos, sorteando una serie de presiones internas, se pronunció por una rápida solución al conflicto. Su postura se vio favorecida por la importancia estratégica del petróleo en la Segunda Guerra Mundial y pudo contrarrestar los embates de los grandes consorcios. Sin el apoyo gubernamental esta. dounidense, la fuerza del bloque se debilitó sensiblemente y la solución al conflicto pasó a ser cuestión de tiempo. Fue así como los intereses norteamericanos que aún permanecían en pugna no pudieron hacer avanzar su posición y tuvieron que replegarse hasta llegar a un acuerdo con el gobierno mexicano respecto del monto de su indemnización, así como de la forma en que se les pagaría, en

24 Ibid., p. 459. 
1943.25 Con ello, la deuda con uno de los colosos, la Standard Oil, estaba próxima a saldarse y el desmantelamiento de la postura de las grandes corporaciones era ya un hecho evidente.

Frente al curso que habian seguido los acontecimientos y bajo el influjo de las alianzas de posguerra, las huestes británicas no tuvieron más remedio que reconocer lo que era ya un hecho y ceder en cuanto a la aceptación de la nacionalización. Apenas concluida la confrontación bélica, las pláticas con el gobierno mexicano -ahora encabezado por Miguel Alemán- pudieron ser reanudadas y avanzar, tras algunos forcejeos, con la Royal Dutch Shell, hasta llegar a un acuerdo definitivo en 1947.26 Los compromisos de alli derivados pudieron finiquitarse en 1962, con lo que se daba carpetazo final al largo y dificil proceso negociador. ${ }^{27}$

Resulta interesante observar al respecto que la claudicación de la postura británica en el conflicto coincide, precisamente, con el debilitamiento del poder monopólico de "las siete hermanas", 28 Concuerda, por tanto, con el inicio del derrumbamiento de lo que se dio en llamar su época de oro, esto es, aquella en la que la hegemonía de las grandes corporaciones se sustentaba en los principios de la secuencia ascendente de la renta diferencial. Ocurre, pues, en un momento en el que las viejas formas de organización monopólica de la industria petrolera internacional comienzan a resquebrajarse y el imperativo de transitar hacia nuevas formas, más acordes con la naturaleza del imperialismo, no puede continuar siendo eludido. Las posturas asumidas por Inglaterra y Estados Unidos en el conflicto se asocian de alguna manera con este hecho. Podríamos visualizarlas como la proyección de dos liderazgos en el seno del imperialismo: uno que había quedado definitivamente atrás y que no lograba desprenderse de sus ataduras con las formas caducas de dominación y otro que

${ }^{25}$ Lorenzo Meyer, México y los Estados Unidos en el conflicto petrolero (1917.1942), 1a. reimpresión, COLMEX, México, 1981, p. 457.

${ }^{26}$ Miguel Alemán, op. cit., pp. 699-741.

27 Samuel I. del Villar, op. cit., p. 125.

28 Este grupo lo conforman cinco empresas norteamericanas (Exxon, Mobil Oil, Standard Oil de California, Gulf Oil y Texaco), una angloholandesa (Royal Dutch Shell) y una inglesa (British Petroleum). 
expresaba el ala avanzada y que fortalecía su comando sobre las nuevas formas de dominación que el propio imperialismo reclamaba.

\section{Los limites de la nacionalización}

Paralelamente al desarrollo del conflicto y fuertemente determinado por las circunstancias que se derivan de él, se produce un vuelco radi. cal en la orientación del crecimiento de la industria petrolera del país. Ésta, por las limitaciones que el boicot le impuso, se vio incapa. citada para seguir mirando hacia el exterior y debio canalizar sus esfuerzos a la satisfacción del mercado interno. Su nueva orientación, que marcaría el carácter de la etapa nacionalizada de la industria hasta la irrupción del boom reciente, implicó toda una redefinición del papel del petroleo en el crecimiento económico del país. Redefinición que haría del valioso energético un símbolo de nacionalismo mexicano: los recursos del país debian ser en adelante exclusivamente para nuestros compatriotas y ya no más para el capital extranjero.

Bajo el nuevo estandarte de la ideología oficial, la funcion de Pemex no podía ser otra que la de apoyar el crecimiento económico del pais, que ahora había dejado su forma orientada al mercado externo e iniciaba su proceso de industrialización sustitutiva. Al no poderle servir de soporte por la vía de las exportaciones, que por lo demás estaban penadas por la nueva ideología, debió hacerlo satisfaciendo e incentivando la demanda doméstica. ${ }^{29}$

La orientación hacia adentro de Pemex, en el contexto de la finali. dad que el Estado le asignaba respecto del crecimiento económico del pais, dio paso a una de las etapas más dificiles de nuestra industria petrolera. Fue una etapa que se caracteriź por los numerosos obstácu-

29 Cabe senalar, sin embargo, que, sin que figurara como su destino principal, "...la industria petrolera pudo exportar en los anos cuarenta en términos netos 24 millones de barriles de crudo para financiar las importaciones de bienes de capital y pagar tam. bién una parte considerable de la deuda negociada con las companías petroleras extranjeras por causa de la nacionalización", Miguel S. Wionczek (coord.), Problemas del sector energético en México, COLMEX, 1983, p. 15. 
los que debieron ser enfrentados para reorientar la industria y sacar adelante la producción sin la tutela de los grandes consorcios, y, en cambio, sí con su bloqueo inicial. Aunque la respuesta que al respecto pudo dar Pemex podría calificarse como satisfactoria, en el sentido de que pudo cubrir en gran medida los requerimientos internos de hidrocarburos, ello se produjo bajo la rúbrica de un ritmo de crecimiento relativamente modesto de la industria nacional. Durante prácticamente toda esta etapa y hasta un año antes de iniciarse el boom reciente, los niveles de la producción no habían podido alcanzar el máximo de 1921 (véase el cuadro 1). O sea que debieron transcurrir casi cuatro décadas y ocurrir el primer choque de petróleo para que Pemex lograra alcanzar las cifras productivas de sus antecesoras extranjeras.

Respecto de los múltiples problemas que en esta etapa enfrentó el crecimiento de Pemex, destaca lo siguiente:

1. Para adecuarse a su nueva finalidad, la industria nacional tuvo que remover dos importantes trabas derivadas de su orientación precedente: "... la ausencia de vías de comunicación más o menos directas entre la mayor parte de los centros de consumo y las refinerías situadas en el Golfo de México, y la escasez y deficiencia del sistema de transporte, particularmente de los ferrocarriles". ${ }^{30}$ La construcción del oleoducto Poza Rica-Azcapotzalco (1946), de la red abastecedora de gas natural a Monterrey (1948) y de las refinerías de Salamanca (1950) y Minatitlán (1956) dan cuenta de los esfuerzos reorganizativos que debieron ser emprendidos en ese sentido ${ }^{31}$

2. Al cúmulo de presiones financieras del aspecto anterior se suma otra, corolario de la finalidad asignada a Pemex por el Estado: el abastecimiento nacional subsidiado de hidrocarburos. Si en la etapa dominada por las empresas extranjeras, el petróleo se vendía internamente a un precio superior al internacional, en ésta, la política de precios buscaría favorecer al mercado nacional. En este sentido, no obstante que los precios internos

${ }^{30}$ Miguel Alemán, op. cit., p. 582.

31 "Cronología de la industria petrolera mexicana", El petróleo en México y en el mundo, 2a. ed., Conacyt, México, 1980, pp. 248 y 249. 
no parecen haber diferido mucho con los vigentes en los Estados Unidos (como lo sugiere la tendencia seguida por los precios de gasolina), ${ }^{32}$ el cambio significó un abaratamiento considerable de los costos de energía en el país. Y algo todavía más importante: debido a las dificultades administrativas, financieras y técnicas por las que atravesaba la industria nacionalizada, dicha política expresaba un verdadero precio subsidiado, esto es, un precio que implicaba una merma progresiva a las finanzas de Pemex, cuestión esta última que no solamente significaba un gravoso sacrificio de sus ganancias potenciales, sino que ponía en jaque el cumplimiento de su principal función: satisfacer la demanda interna.

3. Debido a su papel decisivo para las perspectivas de expansión de la industria, uno de los puntos más débiles y problemáticos de las actividades de Pemex lo fue, sin duda, la limitación de que fueron objeto sus programas de exploración. Como claramente se desprende del cuadro 2 , el coeficiente de reserva sufrió varios desplomes significativos a raíz de la nacionalización, entre los que destacan los ocurridos en 1948, 1950 y 1975. A pesar del desesperado esfuerzo de Pemex por tratar de mantener en un nivel razonable dicho coeficiente, celebrando de 1949 a 1951 cinco contratos-riesgo con empresas norteamericanas indepen. dientes, los avances en este renglón fueron más bien modestos y proyectaban el elevado grado de dependencia tecnológica de la compañía nacional. Los inconvenientes que desde la ideología nacionalista mostraban tales contratos se desprenden de su propio texto, al señalar

... como remuneración por parte de Petróleos Mexicanos el rembolso de los gastos o inversiones por trabajos ejecutados, sin plazo de vencimiento al respecto, con el valor de un porcentaje de la produccion de hidrocarburos que se obtuviera en los pozos perforados y una compensación de 15 a $18.25 \%$ del valor de la producción, por un periodo de veinticinco anos ${ }^{33}$

32 Luis Pazos, Mitos y realidades del petróleo mexicano, 2a. reimpresión, Diana, México, 1979, p. 59.

${ }^{33}$ Miguel Alemán, op. cit. 
MÉxICO: ESTADÍSTICAS BÁSICAS DE LA INDUSTRIA PETROLERA NACIONALIZADA

ANTERIORES AL AUGE DE LOS SETENTA

\begin{tabular}{|c|c|c|c|c|c|c|c|c|}
\hline \multirow{3}{*}{100} & \multicolumn{5}{|c|}{ millowes de Nerriles } & \multirow{3}{*}{ Exportación } & \multirow{3}{*}{$\begin{array}{c}\text { Orapeción } \\
\text { (miles de } \\
\text { trab) }\end{array}$} & \multirow{3}{*}{$\begin{array}{l}\text { Cofficiende } \\
\text { reservav } \\
\text { produccion. }\end{array}$} \\
\hline & \multirow[t]{2}{*}{ Reservas } & \multirow{2}{*}{$\begin{array}{l}\text { Productios } \\
\text { refinados }\end{array}$} & \multicolumn{2}{|c|}{ Exportaciomes } & \multirow{2}{*}{$\begin{array}{l}\text { Imporiacidan } \\
\text { refinedias }\end{array}$} & & & \\
\hline & & & ernate & refinadas & & & & \\
\hline 1938 & 1276 & 34 & 4.0 & 5 & 2 & 23.2 & 17.6 & 32.7 \\
\hline 1939 & 1150 & 42 & 9.0 & 6 & 1 & 33.6 & 20.1 & 27.7 \\
\hline 1940 & 1225 & 32 & 9.0 & 4 & 2 & 28.8 & 21.9 & 27.8 \\
\hline 1941 & 1225 & 35 & 8.0 & 8 & 2 & 35.5 & 19.8 & 28.5 \\
\hline 1942 & 1236 & 33 & 1.0 & 5 & 2 & 17.3 & 20.6 & 35.3 \\
\hline 1943 & 1257 & 34 & 1.0 & 4 & 3 & 16.1 & 21.2 & 35.9 \\
\hline 1944 & 1548 & 36 & 1.0 & 4 & 3 & 12.7 & 22.9 & 41.7 \\
\hline 1945 & 1515 & 43 & 2.0 & 6 & 4 & 18.5 & 25.6 & 34.4 \\
\hline 1946 & 1437 & 44 & 3.0 & 6 & 4 & 18.6 & 29.2 & 29.3 \\
\hline 1947 & 1388 & 51 & 7.0 & 7 & 6 & 24.3 & 28.8 & 24.3 \\
\hline 1948 & 1367 & 50 & 7.0 & 6 & 5 & 21.9 & 29.1 & 22.8 \\
\hline 1949 & 1650 & 55 & 7.0 & 7 & 6 & 22.3 & 29.1 & 26.2 \\
\hline 1950 & 1608 & 56 & 12.0 & 4 & 7 & 22.3 & 34.1 & 21.7 \\
\hline 1951 & 1919 & 61 & 14.0 & 3 & 8 & 21.4 & 36.5 & 24.3 \\
\hline 1952 & 2241 & 65 & 9.0 & 6 & 8 & 19.4 & 35.0 & 28.4 \\
\hline 1953 & 2233 & 71 & 3.0 & 12 & 9 & 20.7 & 36.9 & 30.2 \\
\hline 1954 & 2549 & $n$ & 5.0 & 19 & 11 & 27.3 & 39.9 & 30.0 \\
\hline 1955 & 2751 & 81 & 6.0 & 20 & 15 & 28.3 & 43.3 & 30.2 \\
\hline 1956 & 2959 & 81 & 7.0 & 17 & 18 & 25.1 & 42.5 & 31.5 \\
\hline 1957 & 3374 & 85 & 4.0 & 12 & 17 & 17.4 & 44.4 & 36.7 \\
\hline 1958 & 4070 & 94 & 1.0 & 11 & 12 & 11.6 & 45.5 & 40.3 \\
\hline 1959 & 4348 & 102 & 0.1 & 13 & 8 & 12.5 & 45.7 & 41.0 \\
\hline 1960 & 4787 & 102 & 1.0 & 7 & 8 & 6.9 & 46.7 & 43.9 \\
\hline 1961 & 4990 & 116 & 7.0 & 8 & 7 & 13.0 & 46.2 & 42.6 \\
\hline 1962 & 5008 & 116 & 7.0 & 11 & 6 & 15.1 & 47.4 & 41.0 \\
\hline 1963 & 5150 & 118 & 7.0 & 11 & 7 & 14.8 & 49.6 & 40.9 \\
\hline 1964 & 5227 & 127 & 8.0 & 9 & 10 & 13.2 & 50.4 & 40.5 \\
\hline 1965 & 5078 & 127 & 5.0 & 15 & 9 & 15.0 & 54.0 & 38.5 \\
\hline 1966 & 5357 & 129 & - & 17 & 13 & 12.6 & 57.7 & 39.7 \\
\hline 1967 & 5486 & 147 & - & 18 & 12 & 14.1 & 62.7 & 43.2 \\
\hline 1968 & 5530 & 156 & - & 16 & 12 & 11.6 & 67.7 & 39.8 \\
\hline 1969 & 5570 & 161 & -. & 16 & 17 & 11.1 & 68.4 & 38.1 \\
\hline 1970 & 5568 & 176 & - & 22 & 17 & 14.4 & 71.1 & 35.9 \\
\hline 1971 & 5428 & 178 & - & 17 & 25 & 11.2 & 75.5 & 35.2 \\
\hline 1972 & 5388 & 193 & - & 9 & 25 & 5.8 & 75.7 & 33.5 \\
\hline 1973 & 5432 & 206 & - & 9 & 33 & 5.4 & 76.7 & 33.9 \\
\hline 1974 & 5773 & 234 & 6.0 & 7 & 24 & 6.6 & 77.7 & 30.9 \\
\hline 1975 & 6338 & 241 & 34.0 & 3 & 25 & 14.9 & 81.2 & 25.5 \\
\hline 1976 & 11160 & 268 & 34.0 & 1 & 16 & 12.4 & 88.0 & 38.7 \\
\hline
\end{tabular}

- Exportaciones de crudo y refinados respecto de la producción total de petróleo.

FUENTE: NAFINSA, La economía mexicana en cifras, México, 1981, pp. 65-72. 
Ello dio lugar a que el asunto fuera turnado al Congreso de la nación y que se abriera un debate sobre el apartado sexto de la Ley Reglamentaria del artículo 37 constitucional. Como fruto de las discusiones, en 1960 se hicieron algunas enmiendas a la ley correspondiente, con las cuales quedaría prohibida en definitiva la concertación de los contratos-riesgo, iniciándose asimismo un proceso tendiente a la cancelación de los que se firmaron entre 1949 y $19511^{34}$ Los efectos de tales medidas se dejarían sentir casi de inmediato en el coeficiente de reserva. Después de haber alcanzado su nivel más elevado en 1960, tiende a declinar progre. sivamente hasta alcanzar, en 1975, uno de sus niveles historicos más bajos (comparable sólo con aquéllos mostrados en las vísperas de la instauración de los contratos-riesgo). Todo esto no hace sino revelar el estrecho vínculo a que hicimos mención entre el desarrollo de las actividades exploratorias y la dependencia tecnologica de Pemex.

4. Fuertemente ligada a la última observación y atravesando el conjunto de los problemas que hemos planteado, despunta una dificultad inherente a la naturaleza subdesarrollada de nuestro pais: la ausencia de capacidad propia de desarrollo tecnológico. Sin negar la experiencia y los avances que en ciertos renglones operativos se pudieron lograr gracias a la nacionalización, la situación generalizada de Pemex aparece ceñida por esa dificultad, como claramente se desprende de la siguiente evaluación hecha por Leopoldo García-Colín, ex subdirector del Intituto Mexicano del Petroleo:

Por casi cuarenta anos hemos comprado tecnología petrolera y en la actualidad todavía no somos capaces de copiar los procesos existentes; mucho menos tenemos capacidad para generar tecnología, salvo unos cuantos casos aislados y generalmente simples en su conformacion ${ }^{35}$

34 Ibid., p. 682.

35 Leopoldo García.Colín Sherer. "La ciencia y la tecnología del petróleo: situación actual y perspectivas en México", Las perspectivas del petróleo mexicano, COLMEX, México, 1979, p. 77. 
I podríamos concluir, con el mismo autor:

Para países como el nuestro debe quedar muy claro en la mente de los politicos, los estadistas y los administradores, la diferencia entre ser un usuario de la tecnología a ser un creador de ella; a saber conducir un automóvil, darle servicio y saberlo reparar y ser un experto en el diseño y la construcción de un motor de combustion interna. En la industria petrolera actual somos buenos operadores y reparadores de plantas y procesos que operan en dicha industria, pero diffilmente sabemos crear y disenar dichos procesos. ${ }^{36}$

5. Descontando el carácter restringido que el punto anterior confiere a la significación misma de la nacionalización, es de subrayarse el prácticamente nulo avance de Pemex en términos de productividad. Aunque las comparaciones en este rubro pueden verse un tanto distorsionadas por las cualidades "naturales" de los yacimientos involucrados, una caracteristica distintiva y sobresaliente del crecimiento de Pemex es la manera relativamente excesiva en que su personal se fue expandiendo (véase cuadro 2). Resulta muy ilustrativo al respecto el hecho de que entre 1938 y 1973 el ritmo de crecimiento de la producción (a una tasa anual de $4.1 \%$ ) haya sido ligeramente inferior al del empleo (a una tasa anual de $4.3 \%$ ). Más que una cuestión estrictamente técnica, este crecimiento desproporcionado del personal parece haber obedecido, como lo plantea Lorenzo Meyer, a la política reivindicativa del gobierno mexicano hacia el sindicato petrolero. ${ }^{37}$ Esta política buscaba mantener el control de los trabajadores convirtiéndolos en un grupo relativamente privilegiado de la clase obrera mexicana y solapando las prácticas corruptas de los lideres sindicales. Se trata, ante todo, del resul. tado de un difícil proceso negociador derivado de las fricciones con los trabajadores petroleros que siguieron a la expropiación y en las que estaba explicada la transformación del Estado, del

36 Ibid., p. 81 .

${ }^{37}$ Lorenzo Meyer, "El auge petrolero y las experiencias mexicanas disponibles. Los problemas del pasado y la visión del futuro", Las perspectivas del petróleo mexicano, COLMEX, México, 1979, p. 42. 
antiguo aliado en su lucha contra las compañías extranjeras en nuevo patrón. 38

6. Finalmente, a los diversos problemas señalados, que limitaban el crecimiento de la oferta interna y que dificultaban el cumplimiento de la función de Pemex respecto del consumo nacional, se agregaba otro que hacía aún más grave la situación global: el rápido ritmo al que crecía la demanda de hidrocar. buros. Cimentado en las exigencias del proceso de industria. lizacion sustitutiva por el que atravesaba el país e incentivado, en parte, por la política interna de los precios del petróleo, se propicio un crecimiento de la demanda energética superior al de la economía (a una tasa anual promedio de 10\%). ${ }^{39}$ De este modo, las dificultades para cerrar la brecha entre la demanda $y$ la oferta interna de hidrocarburos fueron acrecentándose, a lo que se sumaban los pocos incentivos que se derivan de la baratura y abundancia relativa del crudo en los mercados inter. nacionales de la época. Todo ello repercutió para que en el pe. riodo 1971-1972 se produjera una fuerte crisis de la oferta interna que convirtio a nuestro país, por primera vez en su his. toria, en importador neto de petroleo. ${ }^{40}$

En gran medida, la crisis en la oferta interna de hidrocarburos de principios de los setenta constituía una manifestación de los límites a los que la etapa de crecimiento hacia dentro de Pemex estaba sujeta. Aunque proyectaba un elevado grado de desarrollo de sus contradicciones internas y dejaba entrever un estado de la empresa pública de fuerte deterioro en sus finanzas, grandes debilidades productivas e inciertas perspectivas de crecimiento no expresaban aún un momento que reuniera condiciones suficientes para el cambio. Más bien, se traducía en una situación de emergencia dentro de la línea general de crecimiento de Pemex; una situación en la que la capacidad produc.

38 Marie-France Prevot-Schapira, "Trabajadores del petróleo y poder sindical en México", Miguel S. Wionczek (coord.), Energía en México. Ensayos sobre el pasado y el presente, COLMEX, México, 1982. p. 146.

${ }^{39}$ Miguel S. Wionczek (coord.), 1983, op. cit., p. 15.

40 lbid., p. 17. 
tiva de la compañía nacional debía ser restituida, de tal modo que le permitiera continuar cumpliendo sus funciones para el crecimiento económico del país. Con esa perspectiva, el gobierno mexicano decidió ampliar los recursos destinados a Pemex y, especialmente, aquellos destinados a sus actividades exploratorias. Los resultados que se obtuvieron fueron sorprendentes, abriendo una etapa muy exitosa de grandes descubrimietos para el país. Buena parte de los nuevos campos petroleros, como Cactus y Sitio Grande, baluartes de la riqueza cretácica de nuestro subsuelo, fueron producto de este esfuerzo y su descubrimiento coincide con el momento en que se producía el primer choque petrolero internacional: $1972-1973 .{ }^{41}$

${ }^{41}$ Samuel I. del Villar, op. cit., p. 134. 